\title{
Tradução, adaptação e exploração de propriedades psicométricas da Escala Interação Trabalho-Família Nijmen (SWING) em uma amostra de professores brasileiros
}

\author{
Mary Sandra Carlotto \\ Pontificia Universidade Católica do Rio Grande do Sul \\ Sheila Gonçalves Câmara \\ Universidade Federal de Ciências da Saúde de Porto Alegre
}

\begin{abstract}
Resumo
Instrumentos que avaliam a relação trabalho-família tendem a enfatizar o conflito entre ambas as instâncias. A Escala Interação Trabalho-Família Nijmen (SWING) avalia a interação trabalho-família considerando a direção e a qualidade da influência. Este artigo analisa a adequação do modelo fatorial original da SWING em uma amostra de 506 professores de escolas públicas municipais de uma cidade da região metropolitana de Porto Alegre, RS. Destes, 91,8\% são mulheres, com idade média de 42,6 anos ( $D P=9) ; 61,4 \%$ são casados e $70,6 \%$ possuem filhos. Foi realizada análise fatorial confirmatória, a qual demonstrou que a distribuição em quatro fatores da escala original (Interferência negativa Trabalho-Família; Interferência negativa Família-Trabalho; Interferência positiva Trabalho-Família; Interferência positiva Família-Trabalho) é adequada para a amostra estudada, com índices satisfatórios de ajustamento e de fidedignidade.
\end{abstract}

Palavras-chave: trabalho; família; medidas; análise fatorial; professores.

\begin{abstract}
Translation, adaptation and exploration of psychometric properties of Survey Work-Home Interaction - Nijmen (SWING) in a sample of Brazilian school teachers. Measures to assess work-family relationship tend to emphasize the conflict between both contexts. The Survey Work-Home Interaction - Nijmen (SWING) evaluates the work-family interaction considering the direction and quality of influence. This article analyses the goodness of fit of the original factor model of SWING to a sample of 506 teachers from public schools in a municipality in the metropolitan region of Porto Alegre, RS. Of these, $91.8 \%$ are women, with a mean age of 42 years and six months $(S D=9) ; 61.4 \%$ are married and $70.6 \%$ have children. It was performed a confirmatory factor analysis which showed that the four factors distribution of the original scale (Negative Work-Home Interaction; Negative Home-work Interaction; Positive Work-home Interaction; Positive Home-work Interaction) is suitable for this study sample, with satisfactory indicators of adjustment and reliability.
\end{abstract}

Keywords: labour; family; measurement; factor analysis; teachers.

\section{Resumen}

Traducción, adaptación y exploración de las propiedades psicométricas de la Escala Interacción Trabajo-Familia Nijmen (SWING). Instrumentos que evalúan la relación trabajo-familia suelen enfatizar el conflicto entre las dos instancias. La Escala Interacción Trabajo-Familia Nijmen (SWING) evalúa la interacción trabajo-familia considerando la dirección y la calidad de la influencia. Este artículo analiza la adecuación del modelo factorial original de la SWING para una muestra de 506 profesores de escuelas públicas de una ciudad de la región metropolitana de Porto Alegre, RS. De estos, $91,8 \%$ son mujeres con edad media de 42 años y seis meses $(D T=9) ; 61,4 \%$ son casados y $70,6 \%$ tienen hijos. Fue realizado un análisis factorial confirmatorio que demostró que la distribución en cuatro factores de la escala original (Interferencia negativa Trabajo-Familia; Interferencia negativa Familia-Trabajo; Interferencia positiva Trabajo-Familia; Interferencia positiva Familia-Trabajo) es adecuada para la muestra en estudio, con índices satisfactorios de ajuste y de fiabilidade.

Palabras-clave: trabajo; família; medidas; análisis factorial; profesores. 
$\mathrm{O}$ $s$ trabalhadores na sociedade contemporânea estão cada vez mais preocupados em gerenciar os conflitos vivenciados na tentativa de atender as duplas demandas e responsabilidades provenientes dos papéis profissional e familiar (Moster \& Oldfield, 2009). A tentativa de conciliar o trabalho e a família torna-se um crescente desafio para os trabalhadores e para as empresas (Costa, 2012).

O trabalho e família são dimensões de um único sistema na vida dos trabalhadores de maneira que o que acontece em um desses âmbitos afeta, invariavelmente, o outro. Por uma via, o envolvimento em uma dimensão pode ser capaz de influenciar positivamente o desempenho na outra e, por outra via, o envolvimento em um papel pode ser responsável por prejuízos no desempenho de outro papel em diferente dimensão (Aguiar \& Bastos, 2013; Geurts et al., 2005). O entendimento da dinâmica da interação entre a vida pessoal e profissional pode auxiliar as organizações no planejamento de políticas e práticas direcionadas ao equilíbrio de ambas as instâncias de vida, permitindo maior eficiência na aplicação de recursos e a criação de um ambiente organizacional mais estável e produtivo (Oliveira, Cavazotte, \& Paciello, 2013).

Bond et al. (2007), em uma revisão sobre diversos instrumentos relativos ao ambiente psicossocial de trabalho, aborda, em um capítulo, diversos instrumentos que avaliam a relação trabalho-família. Esses foram desenvolvidos em diferentes contextos, com amostras de diversos tamanhos e populações variadas, e em diferentes etapas de validação. Apenas o Work Family Conflict and Family Work Conflict Scales, de Netemeyer, Boles e McMurrian (1996) e o Survey Work-Home Interaction - Nijmen (SWING), de Geurts et al. (2005), avaliam de forma bidirecional a relação do conflito. No caso da SWING, essa apresenta, ainda, a possibilidade de interações positivas na relação trabalho-família. Pesquisas sobre a saúde ocupacional e bem-estar enfatizam o lado negativo do bem-estar (Schaufeli \& Bakker, 2004) em detrimento dos aspectos positivos, isto é, a questão sobre como as pessoas conseguem manter um funcionamento positivo e saudável. A SWING é embasada no modelo teórico Esforço-Recuperação, de Meijman e Mulder (1988). Este aborda o processo de recuperação que permite aos trabalhadores reter e repor seus recursos. Nesse sentido, considerando a recuperação, também é importante pensar na forma como os trabalhadores empregam o seu tempo de lazer após o trabalho, para o que a família representa um importante recurso (Geurts \& Sonnentag, 2006). A recuperação do estresse laboral em outras instâncias faz-se importante uma vez que tensões não recuperadas vão se acumulando, trazendo problemas de saúde em médio e longo prazos (Meijman \& Mulder, 1998).

A SWING, portanto, avalia a direção (do trabalho sobre a vida privada e vice-e-versa) e a qualidade da influência (negativa ou positiva) (Geurts et al., 2005). Bond et al. (2007) destacam que a escala é aplicável a todos os trabalhadores, independente de sua situação conjugal ou o fato de ter ou não filhos. Além disso, a escala demonstrou associação significativa com variáveis teoricamente relacionadas, como é o caso de características do cargo/trabalho, características da família e indicadores de saúde e bem estar (Geurts et al., 2005), variáveis importantes para o campo da Psicologia da Saúde Ocupacional (National Institute for Occupational Safety and Health - NIOSH, 2008).

Após sua construção, o modelo teórico e propriedades psicométricas da SWING têm sido confirmadas em diferentes países e profissões em estudos como o de Pieterse e Mostert (2005) com uma amostra de trabalhadores industriais na África do Sul, o de Moreno-Jiménez, Vergel, Muñoz e Geurts (2009), na Espanha, com profissionais de emergência, e de Lourel, Gana e Wawrzyniak (2005), na França, com uma amostra multiprofissional.

Dentre as diversas profissões, a docência tem sido uma das que tem merecido especial atenção no que tange a relação trabalho-família. Na perspectiva de Arroyo (2000), poucos trabalhos se identificam tanto com a vida pessoal do trabalhador como o do professor. O trabalho do professor é altamente exigente, o que compromete o tempo de lazer e convívio familiar (Vasques-Menezes \& Gazzotti, 1999).

Por outro lado, estudos sobre Burnout entre professores demonstram que ter companheiro(a) e/ou filhos é um fator de proteção para a Síndrome (Braun \& Carlotto, 2013; Carlotto, 2011). De acordo com Maslach e Jackson (1985), a família exige uma maior maturidade psicológica e um estilo de vida mais estável. Além disso, a vida familiar exige o desenvolvimento de uma visão diferente do trabalho, especialmente no que se refere à divisão de responsabilidades com outras pessoas. A vida familiar também proporciona mais experiência e desenvoltura para lidar com outras pessoas, o que contribui para o desenvolvimento de habilidades para lidar com adversidades. No campo do ensino, tais vivências cobram maior importância pela necessidade de se relacionar com diferentes públicos.

Para o estudo da interação trabalho-família entre professores, o único instrumento identificado por Bond et al. (2007) foi a Work Family Conflict and Family Work Conflict Scales, de Netemeyer et al. (1996). Esta foi validada no Brasil por Aguiar e Bastos (2013) com trabalhadores em geral e avalia apenas as dimensões de conflito. Há, portanto, escassez de instrumentos e estudos brasileiros relativos à interação trabalho-família, considerando direção e qualidade das interações. Nesse sentido, o presente estudo buscou adaptar e estudar a validade da SWING para a população de professores de escolas públicas de um estado do sul do Brasil.

\section{Método}

\section{Participantes}

A escala foi aplicada em 510 professores, distribuídos em 23 escolas municipais de uma cidade da região metropolitana de Porto Alegre (RMPA). Destes, quatro foram excluídos por haverem completado menos de $90 \%$ do instrumento de pesquisa, sendo a amostra final constituída de 506 participantes. A maioria é composta de mulheres (91,8\%), de estado civil casado $(61,4 \%)$, que possui filhos $(70,6 \%)$ e idade média de 
42 anos e seis meses $(D P=9)$, variando de 18 a 68 anos. A formação de grande parte dos participantes é em nível de pósgraduação. Em média, trabalham na profissão há 18 anos ( $D P$ $=8,9)$ com carga horária de 35 horas $(D P=11,6)$, variando de 08 a 60 horas semanais. Possuem, em média, 80 alunos com uma variação de 11 a 500 alunos. A maioria trabalha somente na escola investigada (78,2\%).

\section{Instrumentos}

Escala de interação trabalho-família - survey work-home interaction. Nijmegen (SWING) de Geurts et al. (2005) Trata-se de uma escala autoaplicável que tem como finalidade avaliar a interação da relação trabalho-família considerando sua direção e qualidade em quatro dimensões: (1) Interferência negativa trabalho-família (ITF-), que avalia o impacto negativo de situações de trabalho no funcionamento familiar (Sinto-me irritado em casa por causa das exigências no trabalho), (2) Interferência negativa Família-Trabalho (IFT-), que mede o impacto negativo de questões familiares na situação de trabalho (Tenho dificuldade em me concentrar no trabalho por estar preocupado com um problema na família), (3) Interferência positiva Trabalho-Família (ITF+), referindo-se a influência positiva de situações de trabalho no funcionamento familiar (Sinto-me mais capaz de interagir com a família, amigos e conjugue por casa do que aprendi no trabalho), (4) Interferência positiva Família-Trabalho (IFT+), caracterizada pelo impacto positivo no funcionamento familiar de situações positivas ocorridas no trabalho (Depois de passar um agradável fim de semana com a família, amigos e cônjuge, sento mais satisfação no trabalho.).

A escala original apresentou alfas de Cronbach satisfatórios: ITF- $=0,84 ;$ IFT- $=0,75 ; \mathrm{ITF}+=0,75 ; \mathrm{IFT}+=0,81$. Sua avaliação é realizada por meio de uma escala de pontuação tipo Likert que varia de 0 a 3: 0 para Nunca; 1 para Algumas vezes; 2 para Muitas vezes; e, 3 para Sempre.

Questionário sociodemográfico e laboral. O instrumento é composto por questões acerca de dados demográficos (sexo, idade, situação conjugal, presença de filhos, formação) e laborais (carga horária semanal, número de alunos atendidos diariamente, tempo de trabalho, trabalhar exclusivamente na escola).

\section{Tradução e adaptação}

O procedimento de validação do instrumento foi autorizado por seus autores, representados pela primeira autora, Sabine Geurts. A partir da versão original, em inglês, a adaptação brasileira da SWING seguiu os padrões metodológicos recomendados pela Comissão Internacional de Testes (ITC) para adaptação adequada de instrumentos a outras culturas (Muñiz \& Hambleton, 2000). Para a tradução foram convidados dois psicólogos do trabalho com domínio do idioma inglês e português do Brasil, brasileiros que residiram no Reino Unido. Foi realizado o processo de tradução e retrotradução e posterior comparação de versões. Na avaliação da equivalência semântica, verificou-se que o significado referencial apresentou bons resultados, tendo em vista que todos os itens obtiveram, nas retraduções entre $90,0 \%$ e $100 \%$ de concordância nesse critério (Reichenheim \& Moraes, 2007).

A versão final em português do Brasil foi submetida a uma testagem piloto, para fins de avaliação semântica, com 37 professores de uma escola privada, não pertencentes à população em estudo. Foi solicitado aos sujeitos que manifestassem aspectos relativos ao entendimento de palavras e enunciados. Nessa etapa não houve necessidade de modificação de nenhum item. Foram também avaliados os coeficientes de consistência interna, pelo método alfa de Cronbach, tendo sido obtidos os seguintes valores: ITF- $=0,89$; IFT- $=0,78$; ITF $+=$ 0,$80 ;$ IFT $+=0,80$.

\section{Procedimentos de coleta e análise de dados}

O instrumento foi aplicado nos meses de agosto e setembro de 2013. Primeiramente, foram realizados contatos com a Secretária de Educação do município, momento em que foi apresentado o objetivo do estudo a fim de obter a autorização e o apoio para a realização da pesquisa. Após, os instrumentos foram aplicados nas escolas em reunião de professores sendo explicado para os participantes tratar-se de uma investigação acadêmica, sem quaisquer efeitos avaliativos individuais e/ou institucionais e que as respostas seriam anônimas e confidenciais.

Uma vez preenchido o instrumento, esse era depositado em um envelope, a fim de garantir o anonimato e a confidencialidade. Foram respeitados os aspectos éticos de acordo com a Resolução 466/12 do Conselho Nacional de Saúde, referente à pesquisa com seres humanos. O estudo possui aprovação do Comitê de Ética em Pesquisa da PUCRS (Parecer: 147.616).

O banco de dados foi digitado e, posteriormente, analisado no pacote estatístico SPSS, versão 17.0. Primeiramente realizaram-se análises descritivas de caráter exploratório a fim de avaliar, no banco de dados, a distribuição dos itens, casos omissos e identificação de extremos. Com o intuito de verificar o modelo de quatro dimensões da SWING, realizou-se uma Análise Fatorial Confirmatória (AFC), método de Máxima Verossimilhança. Os principais valores de referência considerados para a aceitação do modelo foram o CFI $\geq 0,95$ e RMSEA $\leq$ 0,05 (Byrne, 2001; Hooper, Coughlan, \& Mullen, 2008). Foi utilizado o programa estatístico AMOS 17.0. A confiabilidade foi avaliada pelo método do alfa de Cronbach.

\section{Resultados}

\section{Estatística descritiva}

As estatísticas descritivas da SWING podem ser observadas na tabela 1 . As médias mais elevadas correspondem às dimensões de interação positivas. A média mais elevada foi a do item 13 (Após um dia ou semana de trabalho agradável eu tenho vontade de envolver-me em atividades com a família, amigos e cônjuge), que pertence à dimensão ITF+ $(M=2,08)$. As dimensões de interação negativa apresentaram médias menores, 
sendo que o item nove (Sinto-me irritado com problemas familiares e descarrego a frustração nos colegas), pertencente à subescala IFT-, apresentou a menor média $(M=0,27)$.

Em relação à correlação item-total corrigida, a maioria dos itens obteve valores superiores a $r=0,40$. Apenas os itens 13 , pertencente à subescala de ITF+ e o item 18 , da subescala de IFT+, obtiveram correlações inferiores de 0,40. Quanto ao coeficiente de consistência interna, verificou-se que a supressão do item nove (IFT-), do item 13 (ITF+) e dos itens 18 e 22, ambos da subescala IFT+, contribuiria para uma elevação do alfa de Cronbach de suas respectivas subescalas.

Em relação à assimetria, apenas o item nove (IFT-) apresentou valor mais elevado. Os itens 13 (ITF+), 18 e 22 (IFT+) apresentaram pequena assimetria negativa. Os demais 19 itens apresentaram pequena assimetria positiva. Além do item nove, nenhum dos 21 itens restantes apresentou valores que excedessem o intervalo de \pm 1 em assimetria. A subescala IFT- foi a que apresentou maior intervalo, em contraposição à ITF-.

Tabela 1

Estatística Descritiva e Consistência Interna dos Itens e Dimensões da Escala Interação Trabalho-Família Nijmen (SWING) $(n=506)$. RMPA, $R S$, 2013.

\begin{tabular}{lcccc}
\hline \multicolumn{1}{c}{ Subescala/Item } & M(DP) & $\begin{array}{c}\text { Correlação } \\
\text { item-total } \\
\text { corrigida }\end{array}$ & Assimetria & $\begin{array}{c}\text { Alfa } \\
\text { sem item }\end{array}$ \\
\hline Interação trabalho-família negativa $(\boldsymbol{\alpha}=\mathbf{0 , 9 2})$ & $1,21(0,72)$ & & 0,32 & \\
1 & $1,23(0,79)$ & 0,64 & 0,39 & 0,91 \\
2 & $1,12(0,92)$ & 0,71 & 0,37 & 0,91 \\
3 & $1,19(0,84)$ & 0,65 & 0,25 & 0,91 \\
4 & $1,32(0,94)$ & 0,74 & 0,23 & 0,91 \\
5 & $1,26(0,92)$ & 0,80 & 0,22 & 0,90 \\
6 & $1,15(0,98)$ & 0,81 & 0,38 & 0,90 \\
7 & $1,12(0,84)$ & 0,78 & 0,40 & 0,90 \\
8 & $1,30(0,89)$ & 0,74 & 0,25 & 0,91 \\
Interação família-trabalho negativa $(\boldsymbol{\alpha}=\mathbf{0 , 8 4})$ & $0,58(0,52)$ & & 0,99 & \\
9 & $0,27(0,55)$ & 0,50 & 1,04 & 0,85 \\
10 & $0,81(0,65)$ & 0,67 & 0,63 & 0,79 \\
11 & $0,64(0,65)$ & 0,81 & 0,86 & 0,72 \\
12 & $0,58(0,65)$ & 0,70 & 0,93 & 0,77 \\
Interação trabalho-família positiva $(\boldsymbol{\alpha}=\mathbf{0 , 8 4})$ & $1,26(0,65)$ & & 0,46 & \\
13 & $2,08(0,83)$ & 0,30 & $-0,49$ & 0,89 \\
14 & $1,07(0,87)$ & 0,73 & 0,46 & 0,78 \\
15 & $1,00(0,81)$ & 0,80 & 0,46 & 0,76 \\
16 & $1,12(0,83)$ & 0,70 & 0,41 & 0,79 \\
17 & $1,06(0,82)$ & 0,73 & 0,47 & 0,78 \\
Interação família-trabalho positiva $(\boldsymbol{\alpha}=\mathbf{0 , 8 1})$ & $1,47(0,75)$ & & 0,31 & \\
18 & $1,82(0,86)$ & 0,35 & $-0,25$ & 0,83 \\
19 & $1,16(1,07)$ & 0,74 & 0,45 & 0,72 \\
20 & $1,15(1,06)$ & 0,78 & 0,45 & 0,71 \\
21 & $1,29(1,02)$ & 0,71 & 0,24 & 0,74 \\
22 & $1,92(0,92)$ & 0,44 & $-0,44$ & 0,82 \\
\hline & & & & \\
& & &
\end{tabular}

\section{Análise fatorial confirmatória}

Foi testado, inicialmente, um modelo que estabelecia relação entre todos os quatro fatores. No entanto, as relações foram significativas apenas entre os dois fatores positivos e entre os dois negativos $(p=0,001)$. Retirando-se as relações não significativas, a análise apresentou os seguintes índices de adequação do modelo: $X^{2}=642,586(p=0,00) ; X^{2} / g l=3,10$; $\mathrm{TLI}=0,92 ; \mathrm{RMSEA}=0,06$ (IC = 0,06-0,07); CFI = 0,93. A fim de melhorar a adequação, foram empregadas análises e modificações post-hoc (Byrne, 2001; Lei \& Wu, 2007). Os índices de modificação indicaram melhor ajuste do modelo quando relacionados os erros entre os seguintes pares de itens: 18 e 13 (IFT+ e ITF+), 18 e 22 (ambos da subescala IFT+), 16 e 21 (ITF+ e IFT+) e 2 e 8 (ambos da subescala ITF-). Com estes ajustes, o modelo final (Figura 1) alcançou boa adequação, com todos os índices de ajuste considerados: $X^{2}=513,828(p$ $=0,001) ; X^{2} / g l=2,53 ;$ TLI = 0,95; RMSEA =0,05 $(\mathrm{IC}=0,04-$ 0,06); CFI = 0,95 (Hooper, Coughlan, \& Mullen, 2008).

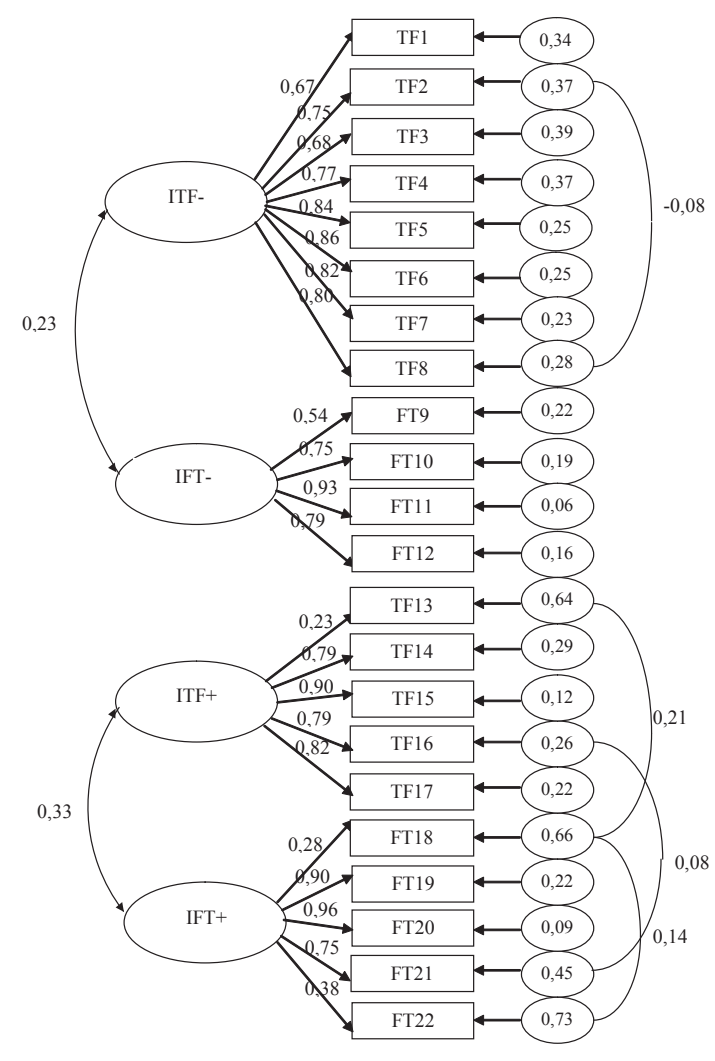

Figura 1. Resultados do Modelo Fatorial Hipotetizado para a Escala Interação Trabalho-Família Nijmen (SWING) $(n=506)$. RMPA, RS. 2013.

\section{Discussão}

O presente estudo objetivou avaliar a estrutura fatorial da SWING em uma amostra de professores, mediante análise fatorial confirmatória. Os resultados corroboraram o modelo hipotetizado de quatro fatores, de acordo com o original da escala holandesa (Geurts et al., 2005), apresentando resultados adequados de validade e confiabilidade para o uso do SWING entre professores no contexto brasileiro. Resultados semelhantes já foram encontrados em outros estudos (Lourel et al., 2005; Moreno-Jiménez et al., 2009; Pieterse \& Mostert, 2005). 
Os valores da correlação item-total corrigida foram elevados, evidenciando que cada dimensão do SWING pode ser considerada com uma função linear dos itens que a compõem. Os valores obtidos para assimetria indicam adequação dos dados. $\mathrm{O}$ valor mais elevado foi identificado no item nove $(A s=$ 1,04) que pertence à dimensão IFT-, o qual não compromete a normalidade. De acordo com Miles e Shevlin (2005), os valores de assimetria aceitáveis estão contidos no intervalo de \pm 1 para que se possa concluir que o modelo tem uma distribuição normal. De acordo com os autores, a assimetria apresentada pelo item nove não compromete a normalidade dos dados. $\mathrm{O}$ item nove também é o que apresenta menor média e correlação item-total corrigida em comparação a todos os demais da escala. Por outro lado, itens com médias mais elevadas (13, 22 e 18), foram os que apresentaram assimetria negativa, dentro do intervalo aceitável.

No que se refere a estes quatro itens, observou-se que sua retirada contribuiria para uma elevação do coeficiente alfa de Cronbach de seus respectivos fatores. Essa elevação, no entanto, não representa um aumento que justifique sua retirada, uma vez que todos os fatores apresentam valores satisfatórios de consistência interna (Tabachnick \& Fidell, 2001). Os valores obtidos em cada dimensão, aliados à coerência semântica do conjunto de itens, permitem considerar que todos contribuem para o estabelecimento do construto ao qual pertencem originalmente no que tange à avaliação da interação trabalhofamília em suas diferentes direções (Geurts et al., 2005).

Desde o estabelecimento do modelo original das escalas, mediante AFC, estudos de validação da SWING em diversos países têm testado a melhor solução fatorial para a mesma, considerando modelos de um, dois, três e quatro fatores. Em todos os casos a conclusão foi que a melhor solução é a de quatro fatores (Geurts et al., 2005; Lourel et al., 2005; MorenoJimenez et al., 2009; Mostert \& Oldfield, 2009).

Neste estudo foi avaliada a versão original da escala (Geurts et al., 2005) e a análise fatorial confirmatória também corroborou a estrutura teórica inicial de quatro fatores. Verificou-se que os fatores positivos e negativos relacionamse entre si e não existe relação entre fatores com qualidades opostas. De acordo com Romeo, Berger, Yepes-Baldó e Belén Ramos (2014), essa configuração não representa prejuízo para a adequação do modelo final e permite uma melhor caracterização de cada um dos quatro fatores em relação à direção e qualidade da interação que avaliam. As correlações encontradas entre as dimensões $(0,23 ; p \leq 0,05$ para as dimensões negativas e 0,$33 ; p \leq 0,05$ para as dimensões positivas) foram inferiores às encontradas por Romeo et al. (2014) (0,61 e 0,72), porém, mais próximas às encontradas na escala original, por Geurts et al. (2005) (0,30 e 0,56).

Todos os itens apresentaram carga fatorial significativa. $\mathrm{O}$ menor valor foi obtido pelos itens $13(\lambda=0,22)$ e $18(\lambda=0,27)$. Nos testes post hoc, os índices de modificação revelaram alta covariância de erro entre os seguintes pares de itens: 13 e 18, 18 e 22, 16 e 21 e 2 e 8 . O item 13 (Após um dia ou semana de trabalho agradável eu tenho vontade de envolver-me em atividades com a família, amigos e cônjuge - ITF+) apresenta o mesmo conteúdo do 18 (Depois de passar um agradável fimde-semana com a família, amigos, conjugue sinto mais prazer no trabalho - IFT+), sendo ambos positivos, com diferença em termos da direção. O item 22 (Sinto mais autoconfiança no trabalho quando tenho a vida familiar bem organizada IFT+) apresenta conteúdo similar ao 18, equivalendo o prazer e autoconfiança no trabalho em função da qualidade na vida familiar. Os itens 16 (Consigo gerir melhor o tempo em casa devido à forma como o faço no trabalho - ITF+) e 21 (Consigo gerir melhor o tempo no trabalho devido à forma como o faço em casa - IFT+) tratam, ambos, da gestão do tempo, também com a diferença na direção. Os itens 2 (Tenho dificuldade em cumprir tarefas domésticas por estar constantemente pensando no trabalho - ITF-) e 8 (O trabalho tira-me tempo que gostaria de passar com a família, amigos, cônjuge - ITF-) também referem-se à gestão do tempo no que diz respeito à interação negativa trabalho-família.

O fato da correlação entre os erros desses pares de itens ter melhorado o ajuste do modelo, aliado às informações da análise de itens, revela que, para a amostra em estudo, a supressão dos itens 13 e 18 contribui para um modelo mais adequado. Esse, sem nenhuma correlação de erro, apresenta os seguintes índices de ajuste: $X^{2}=416,869(p=0,00) ; X^{2} / g l=2,48$; TLI=0,95; RMSEA=0,05 (IC=0,04-0,06); $C F I=0,96$. O presente estudo, no entanto, teve por objetivo uma avaliação inicial do instrumento no Brasil, a partir de sua estrutura original de 22 itens. Nesse sentido, ressalta-se que os diversos aspectos de validade do instrumento apresentaram resultados satisfatórios, indicando que o SWING possui propriedades psicométricas adequadas para o estudo da interação trabalho família entre professores de escolas públicas brasileiros.

$\mathrm{O}$ instrumento pode, portanto, contribuir para a avaliação da importante interrelação entre trabalho e família como um dos aspectos pertinentes à saúde ocupacional docente. Tal avaliação permite subsidiar intervenções de promoção e prevenção nos níveis individual, grupal e institucional.

O estudo apresenta algumas limitações que devem ser consideradas. A primeira é que os participantes são, em sua maioria, do sexo feminino. Dado compatível com a realidade da profissão docente que, tradicionalmente, é exercida predominantemente por mulheres. Esse aspecto é importante, uma vez que homens e mulheres podem diferir em termos de responsabilidades e recursos para estabelecer uma relação mais equilibrada entre o trabalho e família. A segunda refere-se às características contextuais e sociais dos professores investigados. Estes são provenientes de escolas públicas de cidade pertencente à região metropolitana de um estado do sul do Brasil, o que apresenta especificidades em termos da rede de ensino pesquisada e da região do país.

A partir dos resultados encontrados e das limitações do presente estudo, sugere-se que sejam conduzidos novos estudos de validade da SWING com amostra estratificada, a fim de verificar o comportamento do instrumento de acordo com o sexo. Além da avaliação dessa variável, a investigação com trabalhadores de diferentes categorias profissionais, nas diferentes regiões do Brasil, e em diferentes contextos econômicos e 
socioculturais contribuirá para a compreensão da estabilidade do instrumento. Tais abordagens permitirão avaliar, de forma mais consistente, a manutenção ou retirada de itens para sua utilização na realidade brasileira.

De acordo com a proposta do estudo original de validação da SWING, é interessante que estudos possam avaliar as relações da escala com um determinado conjunto de variáveis. Conforme meta-análise realizada por Michel, Kotrba, Mitchelson, Clark e Baltes (2011) sobre o modelo teórico de conflito trabalho-família, estressores de trabalho, envolvimento com o trabalho, apoio social no trabalho e características do trabalho associam-se ao conflito trabalho-família. Já os estressores familiares, apoio familiar e características da família associamse ao conflito família-trabalho. Na interrelação entre estas duas esferas da vida, conforme o modelo de Esforço-Recuperação, as mesmas variáveis podem representar fatores de proteção para o conflito, quando a interação for positiva (Geurts et al., 2005). Nesse sentido, uma importante recomendação para a utilização da SWING para investigar a relação trabalhofamília, é que esta deve vir acompanhada de protocolos que permitam a compreensão da multiplicidade de configurações existentes nas condições de trabalho e no contexto familiar.

\section{Referências}

Aguiar, C. V. N., \& Bastos, A. V. B. (2013). Tradução, adaptação e evidências de validade para a medida de conflito trabalho-família. Avaliação Psicolológica,12(2), 203-212. Recuperado de http://www.redalyc.org/ pdf/3350/335027505011.pdf

Arroyo, M. G. (2000). Ofício de mestre: Imagens e auto-imagens. Rio de Janeiro: Vozes.

Bond, M. A., Kalaja, A., Markkanen, P., Cazeca, D., Daniel, S., Tsurikova, L., \& Punnett, L. (2007). Expanding our understanding of the psychosocial work environment: A compendium of discrimination, harassment, and work-family issues. Washington D.C., USA: National Institute for Occupational Safety and Health. Recuperado de http://198.246.124.22/niosh/docs/2008104/pdfs/2008-104.pdf

Braun, A. C, \& Carlotto, M. S. (2013). Síndrome de burnout em professores de ensino especial. Barbarói, 39, 53-69. Recuperado de http://online.unisc. br/seer/index.php/barbaroi/article/view/2805/3053

Byrne, B. M. (2001). Structural equation modeling with Amos: Basic concepts, applications, and Programming. Nova Iorque: Springer.

Carlotto, M. S. (2011). Síndrome de Burnout em professores: prevalência e fatores associados. Psicologia: Teoria e Pesquisa, 27(4), 403-410. doi: 10.1590/S0102-37722011000400003

Costa, J. C. F. (2012). Práticas de conciliação entre o trabalho e a família: Um estudo exploratório (Dissertação de Mestrado). Universidade do Minho. Braga, Portugal. Recuperado de http://hdl.handle.net/1822/23479

Geurts, S.A.E., \& Sonnentag, S. (2006). Recovery as an explanatory mechanism in the relation between acute stress reactions and chronic health impairment. Scandinavian Journal of Work, Environment \& Health, 32, 482-492. doi: 10.5271/sjweh.1053

Geurts, S. A. E., Taris, T. W., Kompier, M. A. J., Dikkers, J. S. E., Van Hooff, M. L. M., Hooper, D., ... Mullen, M. (2008). Structural equation modeling: Guidelines for determining model fit. Electronic Journal of Business Research Methods, 6(1), 53-60. Recuperado de http://www.ejbrm.com/ volume6/issue 1
Lei, P. W., \& Wu, Q. (2007). Introduction to structural equation modeling: Issues and practical considerations. Educational Measurement: Issues and Practice, 26(3), 33-43. doi: 10.1111/j.1745-3992.2007.00099.x

Lourel, M., Gana, K., \& Wawrzyniak, S. (2005). L'interface «vie privéevie au travail»: Adaptation et validation française de l'échelle SWING (survey work-home interaction-Nijmegen). Psychologie du Travail et des Organisations, 11(4), 227-239. doi: 10.1016/j.pto.2005.10.003

Maslach, C., \& Jackson, S. E. (1985). The role of sex and family variables in burnout. Sex Roles, 12(7/8), 837-851. doi: 10.1007/BF00287876

Meijman, T. F., \& Mulder, G. (1998). Psychological aspects of workload. In P. J. D. Drenth \& H. Thierry (Orgs.), Handbook of work and organizational psychology: Vol. 2. Work psychology (pp. 5-33). Hove, UK: Psychology Press.

Michel, J. S., Kotrba, L. M., Mitchelson, J. K., Clark, M. A., \& Baltes, B. B. (2011). Antecedents of work-family conflict: A meta-analytic review. Journal of Organizational Behavior, 32(5), 689-725. doi:10.1002/job.695

Miles, J., \& Shevlin, M. (2001). Applying regression and correlation: A guide for students and researchers. Londres: Sage Publications.

Moreno-Jiménez, B., Vergel, A. I. S., Muñoz, A.R., \& Geurts, S. (2009). Propiedades psicométricas de la versión española del Cuestionario de Interacción Trabajo-Familia (SWING). Psicothema, 21(2), 331-337. doi: 10.6018/analesps.30.1.148291

Mostert, K., \& Oldfield, G R. (2009). Work-home interaction of employees in the mining industry. The South African Journal of Economic and Management Sciences, 12(1), 81-99. Recuperado de http://www.sajems.org/index.php/ sajems/article/view/262

Muñiz, J., \& Hambleton, R. K. (2000). Adaptación de los tests de unas culturas a otras. Metodología de las Ciencias del Comportamiento, 2(2), 129-149.

Netemeyer, R., Boles, J., \& McMurrian, R. (1996). Development and validation of work-family conflict and family-work conflict scales. Journal of Applied Psychology, 81(4), 400-410. doi: 10.1037//0021-9010.81.4.400

Oliveira, L. B., Cavazotte, F. S. C. N., \& Paciello, R. R. (2013). Antecedentes e consequências dos conflitos entre trabalho e família. Revista de Administração Contemporânea, 17(4)2, 418-437. doi: 10.1590/S141565552013000400003

Reichenheim, M. E., \& Moraes, C. L. (2007). Adaptação transcultural de instrumentos de aferição epidemiológicos: Uma proposta de operacionalização. Cadernos de Saúde Pública, 41, 665-673. doi: 10.1590/ S0102-311X2008000800009

Romeo, M., Berger, R., Yepes-Baldó, M., \& Ramos, B. (2014). Adaptation and validation of the Spanish Version of the "Survey Work-Home Interaction - NijmeGen (SWING) to Spanish speaking countries. Anales de Psicología, 30(1), 287-293. doi: 10.6018/analesps.30.1.148291

Schaufeli, W. B., \& Bakker, A. B. (2004). Job demands, job resources and their relationship with burnout and engagement: A multi-sample study. Journal of Organizational Behavior, 25, 293-315. doi: 10.1002/job.248

Tabachnick, B. G., \& Fidell, L.S. (2001). Using multivariate analysis. (4ª Ed.). Boston: Allyn and Bacon.

Vasques-Menezes, I., \& Gazzotti, A. A. (1999). A si mesmo como trabalho. In W. Codo (Org.), Educação: Carinho e Trabalho (pp. 368-383). Petrópolis: Vozes. 
Mary Sandra Carlotto, Doutora em Psicologia Social pela Universidade de Santiago de Compostela USC, é Professora na Pontifícia Universidade Católica do Rio Grande do Sul (PUCRS). Endereço para correspondência: Programa de Pós-Graduação em Psicologia - Pontifícia Universidade Católica do Rio Grande do Sul . Av. Ipiranga, 6681 - Prédio 11 - Sala 933. Porto Alegre/RS - Brasil -CEP: 90619-900. Telefone: 51-99883637. E-mail: mary.sandra@pucrs.br

Sheila Gonçalves Câmara, Doutora em Psicologia pela Pontifícia Universidade Católica do Rio Grande do Sul (PUCRS), é Professora na Universidade Federal de Ciências da Saúde de Porto Alegre (UFCSPA). E-mail: sheilac@ufcspa.edu.br 